\title{
Evaluation of real-time PCR for the early detection of Legionella pneumophila DNA in serum samples
}

Correspondence

Bram M. W. Diederen

bramdiederen@gmail.com

Received 8 May 2006

Accepted 22 September 2006

\author{
Bram M. W. Diederen, ${ }^{1}$ Caroline M. A. de Jong, ${ }^{1}$ Faïçal Marmouk, ${ }^{1}$ \\ Jan A. J. W. Kluytmans, ${ }^{2}$ Marcel F. Peeters ${ }^{1}$ and Anneke Van der Zee ${ }^{1}$ \\ ${ }^{1}$ Laboratory of Medical Microbiology and Immunology, St Elisabeth Hospital, PO Box 747, \\ 5000 AS Tilburg, The Netherlands \\ 2Laboratory of Microbiology and Infection Control, Amphia Hospital, PO Box 90158, 4800 RK \\ Breda, The Netherlands
}

\begin{abstract}
Legionella pneumonia can be difficult to diagnose. Existing laboratory tests all have shortcomings, especially in the ability to diagnose Legionnaires' disease (LD) at an early stage of the disease in a specimen that is readily obtainable. The aim of this study was to assess the performance of PCR as a rapid diagnostic method and to compare the results of different PCR assays of serum samples from patients with LD. Samples included 151 serum samples from 68 patients with proven LD and 60 serum samples from 36 patients with respiratory tract infections other than

Legionella. PCR assays were based on the 5S rRNA gene, 16S rRNA gene and the mip gene. The samples from patients with infections caused by pathogens other than Legionella all tested negative in PCR. Among the patients with proven LD 54.4\% (37/68) tested positive in 5S rRNA PCR, $52.9 \%$ (36/68) in mip gene PCR and $30.9 \%$ (21/68) in 16S rRNA PCR in the first available serum sample. The association between threshold cycle value in 5S PCR positive serum samples $(n=49)$ and $\mathrm{C}$-reactive protein value was determined, and showed a strong negative correlation (Pearson correlation coefficient $r=-0.63, P<0.0001$ ). In addition to existing tests for the diagnosis of LD, detection of Legionella DNA in serum could be a useful tool for early diagnosis of LD caused by any Legionella species and serogroup, and has the potential to provide a diagnosis in a time frame that could affect initial infection management.
\end{abstract}

\section{INTRODUCTION}

Legionnaires' disease (LD) is an acute pneumonia caused by Legionella spp., which are Gram-negative bacteria ubiquitous in both man-made and natural aquatic reservoirs. Legionella spp. are responsible for 1 to $5 \%$ of cases of community-acquired pneumonia (CAP) (Breiman \& Butler, 1998; Van der Eerden et al., 2005). Although currently 48 Legionella species have been described, more than $90 \%$ of culture-confirmed clinical cases are caused by Legionella pneumophila ( $\mathrm{Yu}$ et al., 2002). Legionella pneumonia can be difficult to diagnose because the signs and symptoms are nonspecific and do not distinguish Legionella infection from other common causes of pneumonia (Den Boer \& Yzerman, 2004). A definite diagnosis is important because it enables adequate antimicrobial treatment; a delay in starting appropriate therapy has been associated with increased mortality (Heath et al., 1996). Currently, the diagnosis of LD in patients with pneumonia is based on phenotypic (culturing, serological testing, antigen detection in urine) and genotypic methods

Abbreviations: CAP, community-acquired pneumonia; CRP, C-reactive protein; Ct, threshold cycle; LD, Legionnaires' disease.
(PCR) using respiratory specimens. Isolation of Legionella from respiratory secretions is considered the gold standard in case definition, but is not very sensitive (10 to $80 \%$ sensitivity) and a positive culture is not normally available until 3 days post incubation (Murdoch, 2003; Den Boer \& Yzerman, 2004). Another major limitation of culturing is that fewer than one half of patients with $\mathrm{LD}$ produce sputum (Murdoch, 2003). Serological testing for Legionella infection is a valuable epidemiological tool but has little impact on clinical decision making because of the time delay before a result is available (Den Boer \& Yzerman, 2004). For the detection of L. pneumophila serogroup 1, urinary antigen tests have sensitivities in the range of $56-99 \%$ and specificities approaching $100 \%$. A major disadvantage with these tests is their inability to reliably detect organisms other than L. pneumophila serogroup 1 (Den Boer \& Yzerman, 2004; Yzerman et al., 2002).

PCR-based methods can detect Legionella-specific DNA in respiratory secretions, with varying degrees of sensitivity and specificity (Murdoch, 2003; Hayden et al., 2001; Cloud et al., 2000; Ballard et al., 2000). Application of PCR to nonrespiratory samples is particularly attractive, because this could circumvent the problem of patients who do not 
produce sputum samples (Diederen et al., 2006; Murdoch et al., 1996). In a recent report, a Legionella specific 5 S rRNA PCR on patient serum was positive in $80.5 \%$ of cases $(54 / 67$ patients), with a peak positivity at 6-10 days after disease onset (Lindsay et al., 2004). Unlike urinary antigen, the number of PCR positives decreased very slowly, perhaps as a result of the slow release of DNA from Legionella surviving in macrophages.

The aim of this study was to assess the performance of PCR as a rapid diagnostic method and to compare the results of different PCR assays in serum samples from patients with LD.

\section{METHODS}

Patients. Samples were collected between June 1999 and May 2005 at the following hospitals in The Netherlands: St Elisabeth Hospital and TweeSteden Hospital, Tilburg; Pasteur Hospital, Oosterhout; TweeSteden Hospital, Waalwijk; Lievensberg Hospital, Bergen op Zoom; Franciscus Hospital, Roosendaal and Amphia Hospital, Breda. The samples were stored at $-70^{\circ} \mathrm{C}$ until they were tested. A proven case of LD was defined as a patient with pneumonia, who showed radiological signs of infiltration, and who showed laboratory evidence of infection with L. pneumophila. Laboratory evidence included (i) a single high value of IgM and/or IgG antibodies or seroconversion for L. pneumophila serogroup 1 to 7 in paired acutephase and convalescent-phase sera using a commercial ELISA (Serion ELISA; Institut Virion\Serion) (Boshuizen et al., 2003), plus (ii) one or more of the following: isolation of L. pneumophila from a respiratory tract sample, a positive urinary antigen test (Binax NOW), a positive PCR result for $L$. pneumophila on a respiratory tract sample using a 16S rRNA assay (Van der Zee et al., 2002).

In addition, 60 serum samples from 36 patients with respiratory tract infections other than Legionella were tested in a similar manner to serve as controls. All control patients tested negative for Legionella antigen in urine (Binax NOW) and L. pneumophila serology (Serion ELISA). Controls consisted of 5 patients with CAP and positive blood cultures with Streptococcus pneumoniae (5 samples) and 31 patients with respiratory tract infections and a fourfold rise in (complementfixating) antibody titre against influenza A virus, adenovirus, Chlamydia psittaci or Mycoplasma pneumoniae (55 samples).

Bacterial strains. Validation of PCR assays was carried out using a panel of human-pathogenic and environmental Legionella spp. The following bacterial strains were used: Philadelphia-1 (SG1) ATCC33152, Togus-1 (SG2) ATCC33154, Bloomington-2 (SG3) ATCC33155, L.A.-1 (SG4) ATCC33156, Dallas-1E (SG5) ATCC33216, Chicago-2 (SG6) ATCC33215, Chicago-8 (SG7) ATCC33823, Concord-3 (SG8) ATCC35096, IN-23 (SG9) ATCC35289, Leiden-1 (SG10) ATCC43283, 797/PA/H (SG11) ATCC43130, 570-CO-H (SG12) ATCC43290, 82A3105 (SG13) ATCC43736, 1169-MN-H (SG14) ATCC43703, Legionella anisa ATCC35292, Legionella birminghamensis ATCC43702, Legionella bozemanii-1 ATCC33217, Legionella bozemanii-2 ATCC35545, Legionella brunensis, Legionella cherii ATCC35252, Legionella dumoffii ATCC35850, Legionella erythra ATCC35303, Legionella feeleii-1 ATCC35072, Legionella feeleii-2 ATCC35849, Legionella gormanii ATCC33297, Legionella hackeliae-1 ATCC35250, Legionella israelensis ATCC43119, Legionella jamestowniensis ATCC35298, Legionella jordanis ATCC33623, Legionella longbeachae-1 ATCC33462, Legionella longbeachae-2 ATCC33484, Legionella maceachernii ATCC35300, Legionella micdadei ATCC33218, Legionella moravica ATCC43877, Legionella oakridgensis ATCC33761, Legionella parisiensis
ATCC35299, Legionella rubrilucens ATCC35304, Legionella sainthelensi ATCC35248, Legionella santicrusis ATCC35301, Legionella spiritensis ATCC35249, Legionella steigerwaltii ATCC35302, Legionella tucsonensis ATCC49180, Legionella wadsworthii ATCC33877. In addition, cultures of non-Legionella bacteria were analysed; Streptococcus pneumoniae ATCC49619, Bordetella pertussis Tohama strain, Bordetella parapertussis B24, Mycoplasma pneumoniae ATCC15293, Chlamydia pneumoniae ATCCVR1355, Acinetobacter baumannii, Pseudomonas aeruginosa ATCC27853 and Lactobacillus casei. In addition, clinical specimens were tested from hospitalized patients with community-acquired respiratory tract infections (Schneeberger et al., 2004) and laboratory evidence for infection with Streptococcus pneumoniae (15), Haemophilus influenzae (10), Moraxella catarrhalis (4), Streptococcus pyogenes (1), Mycoplasma pneumoniae (10) and Chlamydophila pneumoniae (5).

PCR assays. For the detection of Legionella in serum samples three assays were used, targeted at specific regions within the 5S rRNA gene, the 16S rRNA gene and the mip gene (Table 1). The 5S PCR was based on the primers described by Lindsay et al. (2004) and detected in real-time using a TaqMan probe Leg5S. Real-time PCR was performed on an ABI PRISM 7900HT sequence detection system (Applied Biosystems). DNA was amplified according to the following parameters: after $2 \mathrm{~min}$ incubation at $50^{\circ} \mathrm{C}$ and $10 \mathrm{~min}$ denaturation at $95^{\circ} \mathrm{C}$, amplification consisted of 50 cycles of $15 \mathrm{~s}$ denaturation at $95^{\circ} \mathrm{C}$ followed by $1 \mathrm{~min}$ annealing and extension at $60{ }^{\circ} \mathrm{C}$.

DNA extraction. A sample of $200 \mu \mathrm{l}$ was processed with a MagNA Pure LC (Wilson et al., 2004), using the total nucleic acid kit (Roche Diagnostics) with an elution volume of $50 \mu \mathrm{l}$. A $5 \mu$ laliquot of the eluate was used as template in PCR.

Controls. Serum samples from two healthy volunteers were included as negative controls and were processed after every four clinical samples. As an internal control Phocid herpesvirus (PhoHV) was added to the samples to monitor processing as well as inhibition of PCR (Niesters, 2001). Detection of PhoHV was included in the mip gene PCR. In each run a no-template control (mix control) was included. Sensitivity controls for the PCR consisted of 10-fold dilutions of L. pneumophila DNA ranging from 1000 to $10 \mathrm{fg}$ (approximately 230 to 2.3 genome equivalents). Primers and probes were synthesized by Applied Biosystems.

Interpretation of results. Results obtained in real-time PCR were expressed as threshold cycle $(\mathrm{Ct})$ values, corresponding to the cycle at which PCR entered the exponential phase. If no increase in fluorescent signal was observed after 50 cycles, the sample was assumed to be negative.

Statistical analysis. To determine the degree of linear relationship between inflammation [C-reactive protein (CRP) value] and $\mathrm{Ct}$ value, a Pearson correlation coefficient was calculated (http:// statpages.org/).

\section{RESULTS AND DISCUSSION}

Although more than $90 \%$ of culture-confirmed clinical cases of LD are caused by L. pneumophila, some nonpneumophila Legionella species are also known to cause disease in humans (Muder \& Yu, 2002). PCR based diagnosis of Legionella is a very sensitive method, but can be difficult with regard to discrimination of L. pneumophila from other Legionella species. Theoretically, 5S rRNA gene based PCR can detect all Legionella, but the primers have been reported to detect other microorganisms (Maiwald et al., 1995). The mip gene based PCR can detect all $L$. 
Table 1. Primers and probes for PCR assays

\begin{tabular}{|c|c|c|}
\hline Primer/probe & Sequence & Product size \\
\hline \multirow[t]{3}{*}{ 5S rRNA $\mathrm{PCR}^{*}$} & 5S1 (forward 5'-ACTATAGCGATTTGGAACCA-3') & $104 \mathrm{bp}$ \\
\hline & 5S2 (reverse 5'-GCGATGACCTACTTTCGCAT-3') & \\
\hline & Leg5S (5'-FAM-CCGCGCCAATGATAGTGTGAGGC-TAMRA-3') & \\
\hline \multirow[t]{3}{*}{ mip PCR } & Mip-F1 (forward 5'-GCCAAGTGGTTTGCAATACAAA-3') & $80 \mathrm{bp}$ \\
\hline & Mip-R1 (reverse 5'-CTCGACAGTGACTGTATCCGATTT-3') & \\
\hline & LPN-mip (5'-FAM-TAATCAATGCTGGAAATGGTGTTAAACCCG-TAMRA-3') & \\
\hline \multirow[t]{4}{*}{$16 \mathrm{~S}$ rRNA PCR $\dagger$} & Leg-F1 (forward 5'-TACCTACCCTTGACATACAGTG-3') & $150 \mathrm{bp}$ \\
\hline & Leg-R1 (reverse 5'-CTTCCTCCGGTTTGTCAC-3') & \\
\hline & LPN-1 (5'-FAM-AGTCCCCACCATCACAT-MGB-3') & \\
\hline & LSPP-1 (5'-VIC-CCGTAACGAGCGCAA-MGB-3') & \\
\hline \multirow[t]{3}{*}{ PhoHV PCR $\ddagger$} & PhHV-F1 (forward 5'-GGCGAATCACAGATTGAATC-3') & $80 \mathrm{bp}$ \\
\hline & PhHV-R1 (reverse 5'-GCGGTTCCAAACGTACCAA-3') & \\
\hline & PhHV-1 (5'-VIC-TTTTTTATGTGTCCGCCACCATCTGGATC-TAMRA-3') & \\
\hline
\end{tabular}

FAM, 6-carboxyfluorescein; MGB, minor groove binding protein (non-fluorescent); TAMRA, 6-carboxytetramethylrhodamine.

*Primers according to Lindsay et al. (2004).

$\dagger$ Primers according to Van der Zee et al. (2002).

$\ddagger$ Primers according to Niesters (2001).

pneumophila but also may detect some non-pneumophila Legionella species. The 16S rRNA gene based PCR may provide the most clear discrimination between L. pneumophila and other Legionella species (Van der Zee et al., 2002) although its theoretical specificity is no longer $100 \%$ due to the ongoing expansion of sequence data available in recent years. In this study the three PCR assays were revalidated. The analytical specificities and sensitivities of PCR assays were investigated as described in Methods. With the $5 \mathrm{~S}$ rRNA gene based PCR only Legionella species were detected. With the mip gene based PCR all serogroups of $L$. pneumophila were detected and L. israelensis. With $16 \mathrm{~S}$ rRNA based PCR only L. pneumophila and Legionella species were detected; a $100 \%$ specificity of discrimination was observed. No PCR positives were found among nonLegionella bacteria tested. In clinical materials from patients with CAP of known aetiology no Legionella spp. DNA was detected in any of the three assays. The sensitivity of the $5 \mathrm{~S}$ rRNA based PCR assay was highest, with a lower detection limit of $10 \mathrm{fg}$ chromosomal DNA. In the mip gene based assay, the lower detection limit was $100 \mathrm{fg}$, and $1000 \mathrm{fg}$ was found as lower detection limit for the 16S rRNA based PCR.

In order to study the usefulness of serum for PCR-based diagnosis, we included 68 patients with proven LD, with 151 serum samples taken in total. The cases included were 68 adults (48 males and 20 females, male-female ratio 2.4) between 32 and 78 years old (mean age 54.0 years). The laboratory results of these patients were as follows: [positive/ number tested (\%)] serology 68/68 (100\%), isolation 15/29 (52\%), PCR 26/26 (100\%) and urinary antigen test 55/60 ( $92 \%)$. Since no records were made of the first day of illness, we arbitrarily designated the 'acute-phase' serum sampling as the starting point of the disease (day 0). Among the patients with proven LD $54.4 \%(37 / 68)$ tested positive in $5 \mathrm{~S}$
rRNA PCR, 52.9\% (36/68) in mip gene PCR and 30.9\% (21/ $68)$ in $16 \mathrm{~S}$ rRNA PCR in the first available serum sample. Among LD cases, 20/68 (29.4\%) tested positive in all three PCRs (Table 2), with mean Ct values of 29.3, 31.5 and 32.5, respectively, for $5 \mathrm{~S}$ rRNA, mip and $16 \mathrm{~S}$ rRNA gene based PCRs. Another 15 (22.1\%) patients were PCR positive in $5 \mathrm{~S}$ rRNA and mip gene based PCR (mean Ct values of 35.2 and 37.8 ) but negative in $16 \mathrm{~S}$ rRNA based PCR, 1 patient $(1.5 \%)$ was positive in both $5 \mathrm{~S}$ rRNA and $16 \mathrm{~S}$ rRNA based PCR (Ct values of 35.4 and 45.0 , respectively), 1 patient was positive only in 5S rRNA based PCR (Ct value 36.9) and 1 patient was positive only in mip based PCR (Ct value 38.5 ). The detection rate for all (non-inhibited) samples was 34.9\% (49/149), $35.6 \%$ (48/149) and $16.8 \%$ (25/149) for 5S rRNA, mip gene and $16 \mathrm{~S}$ rRNA based PCR, respectively. In two samples (obtained from two patients) inhibition of PCR occurred.

Failure to diagnose and treat LD early has been found to contribute to increased morbidity and mortality (Heath et al., 1996). Existing laboratory tests lack sensitivity in detecting all cases of LD or provide only a retrospective diagnosis. The theoretical advantages of PCR for the detection of L. pneumophila DNA in serum are evident: serum samples are readily obtainable and can be processed within a working day. Our findings are largely in concordance with results previously reported (Diederen et al., 2006). For an illness of low prevalence such as LD the specificity of a test is an important parameter, and our results suggest that the positive predictive value obtained using PCR on serum is high. In contrast to the high sensitivity found by Lindsay et al. (2004), the sensitivity of PCR found in our study was relatively low. Sensitivity of PCR is likely to increase when testing samples that are obtained early in the course of illness, when testing of more 
Table 2. Data for patients with LD

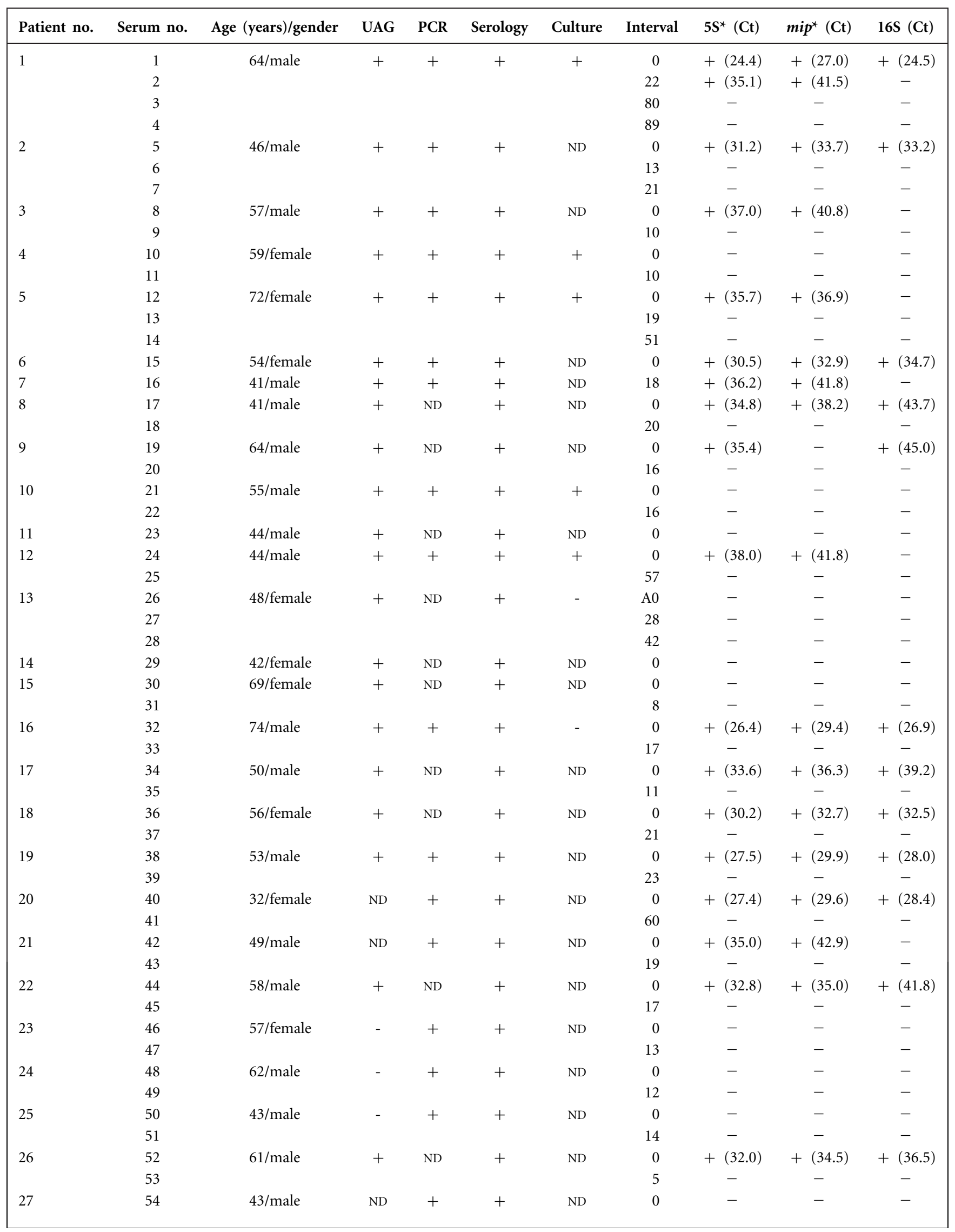


Table 2. cont.

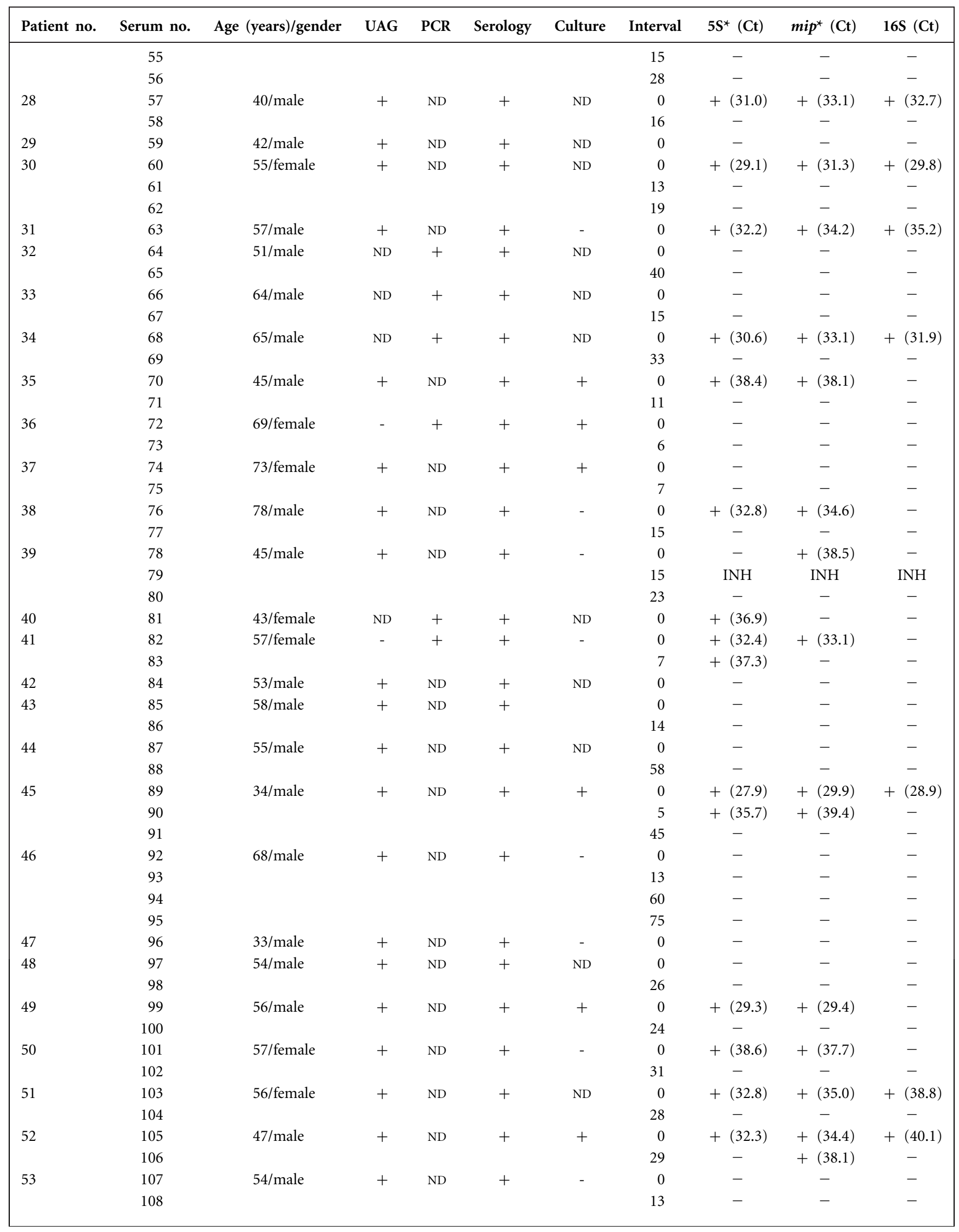


Table 2. cont.

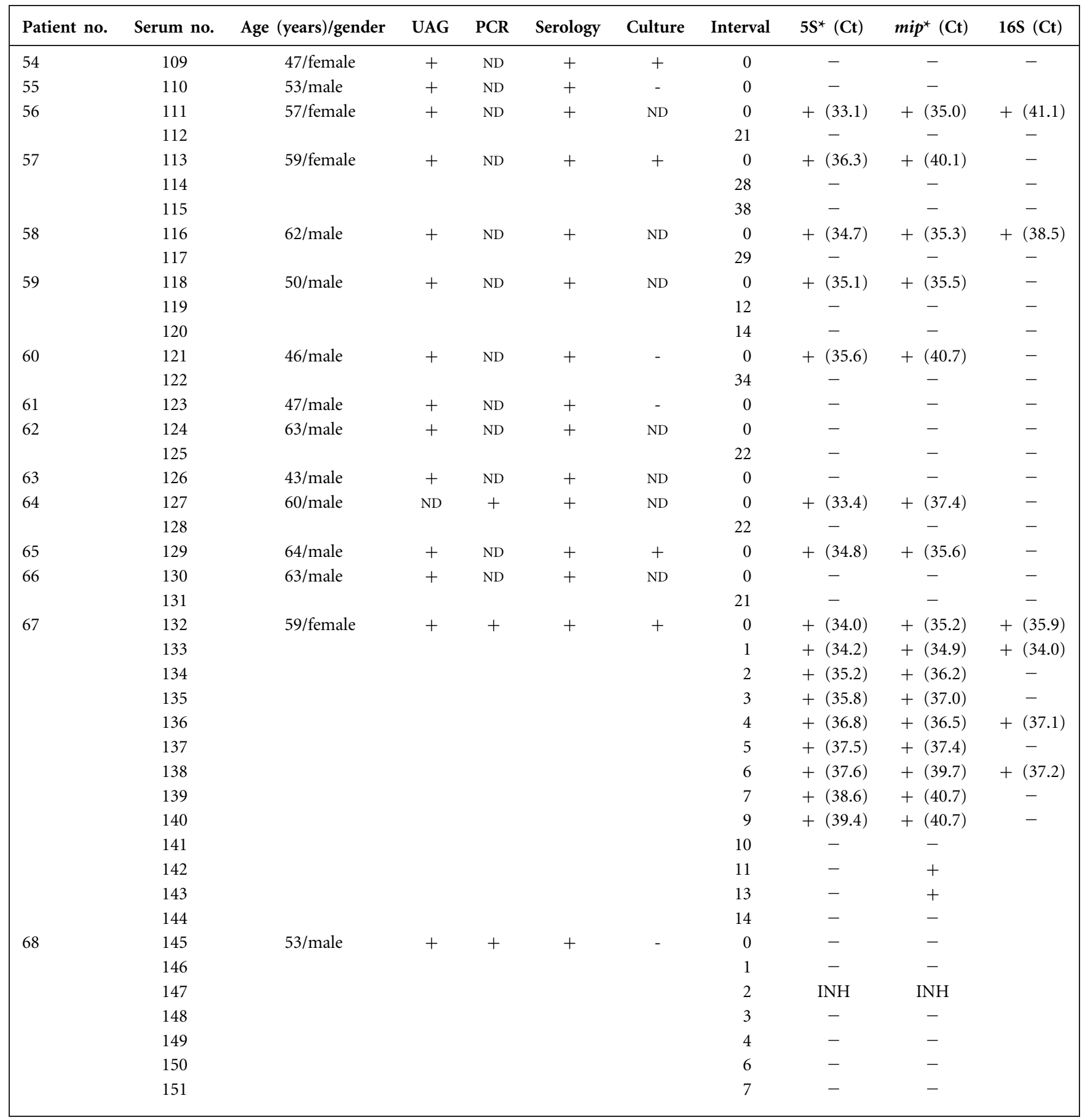

UAG, Urinary antigen test; interval, number of days since first serum sample; INH, inhibition of PCR; ND, not determined.

${ }^{*}$ Among the patients with proven LD $54.4 \%$ (37/68) tested positive in 5S rRNA PCR, 52.9\% (36/68) in mip gene PCR and 30.9\% (21/68) in $16 \mathrm{~S}$ rRNA PCR in the first available serum sample. The detection rate for all (non-inhibited) samples was 34.9\% (49/149), 35.6\% (48/149) and $16.8 \%$ (25/149) for 5S rRNA, mip gene and 16S rRNA based PCR, respectively. In two samples (obtained from two patients) inhibition of PCR occurred.

than one serum sample from each patient is performed and in patients with severe pneumonia, e.g. those with a pneumonia severity index (PSI) of 4 and 5. The urinary antigen test is less reliable in milder cases of LD, and it would be plausible that the same holds true for the detection of Legionella DNA in serum (Yzerman et al., 2002). Because of the retrospective nature of our observations we were not able to investigate the relation between test sensitivity and 
the severity of the disease. However, the presence of $L$. pneumophila DNA in serum could correlate with an acute phase response as reflected in high CRP values (Diederen et al., 2006). The Ct value is inversely proportional to the log of the amount of target DNA initially present. In the current study, the association between $\mathrm{Ct}$ value in $5 \mathrm{~S}$ PCR positive serum samples $(n=49)$ and CRP value was determined, and showed a strong negative correlation (Pearson correlation coefficient $r=-0.63, P<0.0001)$. Wever et al. (2006) studied the kinetics of $L$. pneumophila DNA in serum samples of patients with LD in relation to CRP using a mip gene based PCR. Fifteen hospitalized patients with LD were studied on the day of admission up to 10 days thereafter. Serum samples obtained on the day of admission were mip gene PCR positive in $7 / 11(64 \%)$ patients and negative in 4/11 (36\%) patients. Interestingly, the lowest Ct value (26.6) was found in a patient who died on day 5 following admission. On the day of admission, the mean CRP level in the 7 patients with a mip PCR positive serum sample was $499 \pm 144 \mathrm{mg} \mathrm{l}^{-1}$ (median $\pm \mathrm{SD}$ ) and $244 \pm 97 \mathrm{mg}$ in the 4 patients with a negative PCR result.

Currently, a positive culture, a positive urinary antigen test or a fourfold or greater rise in antibody titre against $L$. pneumophila is definitive of a confirmed case, and PCRpositive samples are only classified as presumptive by the European Working Group on Legionella Infections (EWGLI). There is an apparent need for prospective studies to determine the exact sensitivity and specificity of PCR in patient samples. In our experience, PCR as a diagnostic in patients with severe CAP is a sensitive and specific tool in those who produce sputum. The high specificity is exemplified by a recent investigation conducted by our laboratory (Diederen et al., 2005). In this case-control study, nose and throat samples from patients presenting with acute respiratory tract infections and matched controls were analysed for the presence of Legionella spp. by real-time $16 \mathrm{~S}$ rRNA based PCR. Legionella DNA was not detected in any of the cases or controls. Although our definition of a proven case was different from that defined by EWGLI and included a positive PCR, the majority $(97 \%, 65 / 67)$ of our patients would also be considered a proven case according to EWGLI criteria (Table 2). We performed a retrospective, laboratory-based study, using serum samples from patients with proven LD and patients with respiratory tract infections other than Legionella. Ideally, a prospective study should be conducted to evaluate the value of Legionella-specific PCR using consecutive serum samples in patients with (severe) pneumonia. However, our findings suggest that detection of L. pneumophila DNA in serum could be a valuable tool in addition to existing diagnostic tests for the rapid diagnosis of $\mathrm{LD}$ in the acute phase of disease, and has the potential to detect infections caused by any Legionella species and serogroup. The strong association between $\mathrm{Ct}$ value and CRP value suggest that application of PCR for the detection of Legionella should especially be considered in patients with severe pneumonia of unknown origin. In our view, the best initial testing strategy that will detect all Legionella species and provide results within a time frame that will affect clinical management consists of a urinary antigen test combined with Legionella PCR on sputum and serum.

\section{REFERENCES}

Ballard, A. L., Fry, N. K., Chan, L., Surman, S. B., Lee, J. V., Harrison, T. G. \& Towner, K. J. (2000). Detection of Legionella pneumophila using a realtime PCR hybridization assay. J Clin Microbiol 38, 4215-4218.

Boshuizen, H. C., Den Boer, J. W., de Melker, H., Schellekens, J. F., Peeters, M. F., van Vliet, J. A. \& Conyn-van Spaendonck, M. A. (2003). Reference values for the SERION classic ELISA for detecting Legionella pneumophila antibodies. Eur J Clin Microbiol Infect Dis 22, 706-708.

Breiman, F. R. \& Butler, J. C. (1998). Legionnaires' disease: clinical, epidemiological, and public health perspectives. Semin Respir Infect 13, 84-89.

Cloud, J. L., Carroll, K. C., Pixton, P., Erali, M. \& Hillyard, D. R. (2000). Detection of Legionella species in respiratory specimens using PCR with sequencing confirmation. J Clin Microbiol 38, 1709-1712.

Den Boer, J. W. \& Yzerman, E. P. F. (2004). Diagnosis of Legionella infection in Legionnaires' disease. Eur J Clin Microbiol Infect Dis 23, 871-878.

Diederen, B. M. W., de Jong, C. M. A., Aarts, I., Peeters, M. F., van Gageldonk-Lafeber, A. B., Wilbrink, B. \& Van der Zee, A. (2005). No evidence of Legionella infection in general practice patients presenting with acute respiratory infections in The Netherlands. Clin Microbiol Infect 11, 410-412.

Diederen, B. M. W., de Jong, C. M. A., Kluytmans, J. A. J. W., Van der Zee, A. \& Peeters, M. F. (2006). Detection and quantification of Legionella pneumophila DNA in serum: case reports and review of the literature. J Med Microbiol 55, 639-642.

Hayden, R. T., Uhl, J. R., Qian, X., Hopkins, M. K., Aubry, M. C., Limper, A. H., Lloyd, R. V. \& Cockerill, F. R. (2001). Direct detection of Legionella species from bronchoalveolar lavage and open lung biopsy specimens: comparison of LightCycler PCR, in situ hybridization, direct fluorescence antigen detection and culture. J Clin Microbiol 39, 2618-2626.

Heath, C. H., Grove, D. I. \& Looke, D. F. (1996). Delay in appropriate therapy of Legionella-pneumonia associated with increased mortality. Eur J Clin Microbiol Infect Dis 15, 286-290.

Lindsay, D. S., Abraham, W. H., Findlay, W., Christie, P., Johnston, F. \& Edwards, G. F. (2004). Laboratory diagnosis of Legionnaires' disease due to Legionella pneumophila serogroup 1: comparison of phenotypic and genotypic methods. J Med Microbiol 53, 183-187.

Maiwald, M., Schill, M., Stockinger, C., Helbig, J. H., Luck, P. C., Witzleb, W. \& Sonntag, H. G. (1995). Detection of Legionella DNA in human and guinea pig urine samples by the polymouse chain reaction. Eur J Clin Microbiol Infect Dis 14, 25-33.

Muder, R. R. \& Yu, V. L. (2002). Infection due to Legionella species other than L. pneumophila. Clin Infect Dis 35, 990-998.

Murdoch, D. R. (2003). Diagnosis of Legionella infection. Clin Infect Dis 36, 64-69.

Murdoch, D. R., Walford, E. J., Jennings, L. C., Light, G. J., Schousboe, M. I., Chereshsky, A. Y., Chambers, S. T. \& Town, G. I. (1996). Use of the polymerase chain-reaction to detect Legionella DNA in urine and serum samples from patients with pneumonia. Clin Infect Dis 23, 475-480.

Niesters, H. G. (2001). Quantitation of viral load using real-time amplification techniques. Methods 25, 419-429. 
Schneeberger, P. M., Dorigo-Zetsma, J. W., Van der Zee, A., van Bon, M. \& van Opstal, J. L. (2004). Diagnosis of atypical pathogens in patients hospitalized with community-acquired respiratory infection. Scand J Infect Dis 36, 269-273.

Van der Eerden, M. M., Vlaspolder, F., de Graaff, C. S., Groot, T., Jansen, H. M. \& Boersma, W. G. (2005). Value of intensive diagnostic microbiological investigation in low- and high-risk patients with communityacquired pneumonia. Eur J Clin Microbiol Infect Dis 24, 241-249.

Van der Zee, A., Verbakel, H., de Jong, C., Pot, R., Bergmans, A., Peeters, M., Schneeberger, P. \& Schellekens, J. (2002). Novel PCR-probe assay for detection of and discrimination between Legionella pneumophila and other Legionella species in clinical samples. J Clin Microbiol 40, 1124-1125.

Wever, P. C., van de Veerdonk, F., Schellekens, J. J. A., Huijsmans, C. J. J., de Jager, C. P. C. \& Hermans, M. H. A. (2006). Presence of Legionella pneumophila DNA in serum samples during Legionnaires' disease in relation to C-reactive protein levels. Ned Tijdschr Med Microbiol 14 supplement, S87.

Wilson, D., Yen-Lieberman, B., Reischl, U., Warshawsky, I. \& Procop, G. W. (2004). Comparison of five methods for extraction of Legionella pneumophila from respiratory specimens. J Clin Microbiol 42, 5913-5916.

Yu, V. L., Plouffe, J. F., Pastoris, M. C., Stout, J. E., Schousboe, M., Widmer, A., Summersgill, J., File, T., Heath, C. M. \& other authors (2002). Distribution of Legionella species and serogroups isolated by culture in patients with sporadic community-acquired legionellosis: an international collaborative study. J Infect Dis 186, 127-128.

Yzerman, E. P., Den Boer, J. W., Lettinga, K. D., Schellekens, J., Dankert, J. \& Peeters, M. (2002). Sensitivity of three urinary antigen tests associated with clinical severity in a large outbreak of Legionnaires' disease in the Netherlands. J Clin Microbiol 40, $3232-3236$ 